\title{
Electro-magnetic Sensing and Actuation Array on Silicon Substrate Platforms
}

\author{
Faisal T. Abu-Nimeh and Fathi M. Salem
}

\begin{abstract}
Electromagnetic arrays can be precisely constructed on silicon CMOS technology as a platform for collective non-contact sensing and manipulation of magnetic or magnetized particles of micron to nano scale. Such platforms become a tool to interact with nano scale particles on the silicon substrate and enable direct interface to measurement and computing devices. This paper overviews current efforts along this direction and presents an example design of a platform for sensing and manipulating magnetized beads on its surface using standard silicon CMOS technology. The vision is that such arrays can be easily commanded in nano seconds to realize magnetic field profiles in order to steer magnetized material in the proximity of the platform. Moreover, as technology features decrease, the size of the electromagnetic coil cells in the array would also decrease to nano scale, sharpening the sensing and manipulation resolution of the platforms.
\end{abstract}

\section{INTRODUCTION}

The tools and devices to sense, interact, and manipulate nano-scale elements require precision and efficient interface to electromagnetic computing and displays. The paper focuses on silicon CMOS technology as an enabler technology for tools and devices to sense and control nano-scale elements. As an example, it describes the development of silicon substrate integrated electromagnetic coil arrays for sensing and actuation of magnetic or magnetized beads or elements. This example design illustrates the state-of-the-art in this field and indicates directions of further investigations to realize platforms for sensing and controlling nano-scale material in real-time interaction in the order of nano-seconds. Other design examples addressing the applications of electromagnetic arrays have focused on bio-material and can be found in, e.g., [1]-[4].

\section{System DESIGN}

Without relying on any external magnets it is possible to create localized high-resolution electromagnetic fields using on-chip coil arrays. The size of the coil, the number of layers used, and the shape of the array are often mandated by (i) the application of interest and (ii) the CMOS technology at hand. Additionally, it is possible to share the same coil for sensing and actuation [1], however, there are trade-offs to be considered in order to design the array for both modes. The remainder of this paper focuses on the actuation example which requires challenging designs. As for the sensing and shared sensing and actuation, we refer the reader to the example designs in [1]. In this paper, we describe the design

Circuits, Systems, and Neural Networks Lab, Dept. of Electrical \& Computer Engineering, Michigan State University, East Lansing, USA $\{$ asd1815, salem\}eegr.msu.edu

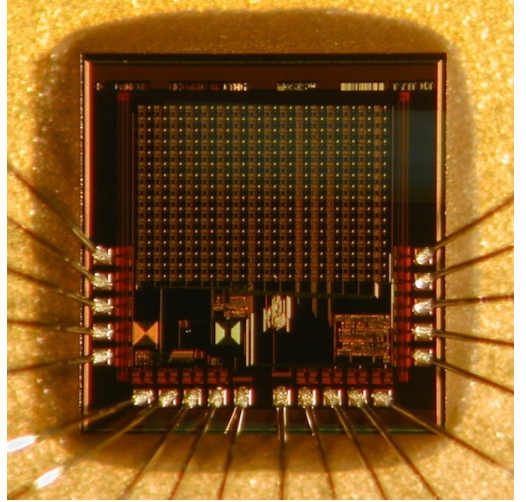

Fig. 1. Silicon-die of an integrated 16x16 array of coil actuators with support circuitry.

of an 8x8 magnetic manipulation coil-array suitable for bio-material applications in standard $0.5 \mu \mathrm{m}$ CMOS. This design builds on a completed similar design for a 16x16 coil array platform, depicted in the die image in figure 1, which was tailored for low-force applications [1]. In the present design, where more power and consequently more force is desired, the size of the $8 \times 8$ array platform is in the order of $248 \mu \mathrm{m} \times 248 \mu \mathrm{m}$. A single cell (coil) occupies $30 \mu \mathrm{m} \times 30 \mu \mathrm{m}$ and the distance between any two coils is $0.9 \mu \mathrm{m}$ which is the smallest DRC (Design Rule Checking) value permissible in this CMOS technology. As a result of making the coil cell elements of the array closely stacked, by moving digital switches and controllers to the sides, one can form an arbitrary number of magnetic field profiles (or forces) using collaborative actuation across the array. The system-level block diagram shown in figure 2 consists of four major components; the $8 \times 8$ coil array, 3 -to- 8 row and column decoders, a variable power bi-directional current source, and the global control. First, the global control is responsible for: (a) coordinating the magnetic fields generated by providing the correct signals to the decoders and the current source. (b) providing a flexible interface to allow programmable off-chip algorithms. (c) fast switching with a period of $25 \mathrm{n}$ seconds for pseudo-parallel operation. Second, the row/column decoders and digital logic facilitate single coil selection and minimize the overall number of I/O pins of the package. Third, an eight-level bi-directional current source to generate different magnetic flux densities and directions for refined maneuvering. Lastly, the coil array where the electric current is passed through a selected coil location to generate an electromagnetic field using on-chip multi-layered inductors. 


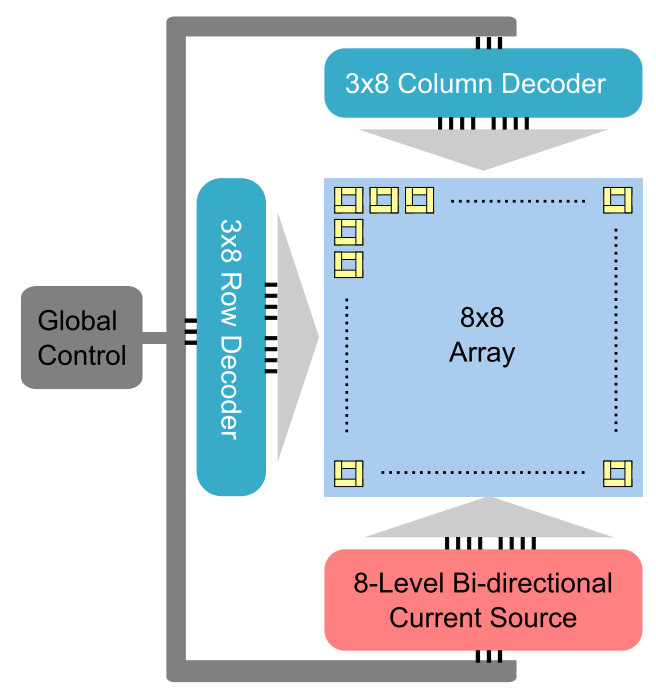

Fig. 2. System-level block diagram.

All of the aforementioned components are integrated on the same die and fabricated using standard CMOS technology then packaged using standard ceramic or plastic packages. Contrary to other state-of-the-art implementations, our method does not require any special post-processing such as microfluidic structures to channel or manipulate bio-materials. It is designed to operate as an "open cavity" device where the entire surface of the chip is exposed to the sample or material of interest. This reduces the complexity of the system, makes it cheaper to manufacture, and more portable by eliminating microfluidic structures overhead and the micro-pumps associated with it. However, it introduces new challenges, in which, some are shared with microfluidic structures. The challenges are mainly in terms of packaging: insulating the wire bonds attaching the CMOS die with the ceramic/plastic package, biocompatibility of the surface of the die, and maneuvering the sample of interest in open space.

Careful design, integration, and packaging of all components is due in order to precisely manipulate bio-materials in the order of $1 \mu \mathrm{m}$ in diameter for this $0.5 \mu \mathrm{m}$ CMOS technology. However, for sub-micron object manipulation one can select a smaller CMOS technology e.g. $45 \mathrm{~nm}$ in order to manipulate smaller objects. The following sections expand on the design, packaging, and experimental results.

\section{Specifications ANd Considerations}

The application of interest specifies the general guidelines for the system design and assembly. For example, the amount of magnetic force required for manipulation, the ambient temperature of operations, the shape of the array, etc., depend on the targeted material. Next, we discuss some of these specifications and limitations.

\section{A. Manipulation Forces}

In this design example, the magnetic forces generated by a single coil should be capable of moving a single magnetic (or magnetized) bead suspended in water or saline. Assuming room temperature operation and a stationary setup, there are mainly two forces influencing the magnetic bead [3]:

$$
\begin{aligned}
\vec{F}_{m} & =\frac{1}{2 \mu_{0}} \Delta \chi V_{m} \nabla \vec{B}^{2} \\
\vec{F}_{d} & =-3 \pi D_{h} \eta \vec{v}_{s},
\end{aligned}
$$

where $\overrightarrow{F_{m}}$ is the magnetic force generated on the bead, $\mu_{0}$ is vacuum permeability, $\Delta \chi$ is the difference in the relative magnetic susceptibility between the magnetic bead and the medium around it, $V_{m}$ is the volume of the bead, and $\nabla \vec{B}^{2}$ is the gradient of the (norm squared of the) magnet flux density. Also, $\vec{F}_{d}$ is the drag force acting against the movement, $D_{h}$ is the hydraulic diameter of the bead, $\eta$ is the dynamic viscosity of the fluid, and $\overrightarrow{v_{s}}$ is the velocity of the bead relative to the fluidic environment.

There are only three parameters to adjust in the equations above; the type of the magnetic bead which is typically $1 \mu \mathrm{m}$, to $8 \mu \mathrm{m}$ in diameter with a known volume and magnetic susceptibility. Second, the medium surrounding the beads might be the sample of interest itself, e.g. blood samples, or biological cell cultures suspended in a medium. Third, the magnetic flux density generated by the integrated coil array. Therefore, if the magnetic bead and the medium surrounding the biological sample are already known, the only parameter to be set is $\vec{B}$ in order to generate the forces of interest. In this work, the magnetic forces ranging from $10 \mathrm{pN}$ to $6 \mathrm{nN}$ are considered sufficient for manipulation [4], [5].

\section{B. Electric Current}

The electric current passing through a coil creates a magnetic field which is more concentrated in the center of coil. Therefore, the magnetic force generated by a coil is proportional to the magnetic flux density and the current as $F_{m} \propto B^{2} \propto I^{2}$. As a result, to generate larger forces one needs to drive more current through the coil. However, increasing the current inadvertently will cause the coil to heat up which may damage the interconnects/via in the CMOS platform itself in addition to damaging the sample of interest.

\section{Shape of Coil and Array}

The shape of the coil, and thus its magnetic flux density and force, can be synthesized using the number of turns, the spacing between turns, the width of the metal in each turn, and the number of planar metal layers stacked on top of each other. The type of metal and the distance between layers are often enforced by the technology and the fabrication process. Additionally, the standard CMOS technologies used here do not support Non-Manhattan shapes like circular or spiral coils. Therefore, we are limited to use square shapes for horizontal and vertical routing. This produces challenges in terms of the generated shape of the magnetic flux density and also in the approximating equations used to model the forces. Determining the optimal coil shape to create a desired force is a subject of future publications, however, by using the smallest allowable DRC metal width and spacing one 
can achieve acceptable actuator units in an array. The only constraint which limits the force in this case is the maximum current density specified by the process for that CMOS technology. The current density $J$ is defined as

$$
J=\frac{I}{W},
$$

where $I$ is the current in Amperes and $W$ is the width of the metal in Meters. For example, if the maximum current density for "via" in a CMOS is $1 \mathrm{~mA}$ /unit, the maximum generated force by the coil is limited by $1 \mathrm{~mA}$. If larger forces are required, one has to design a coil with wider metals and also more parallel "via's" or contacts between different metal layers.

The shape of the array is mainly dependent on the targeted object size and the number of metal layers available for routing and interconnects. The object of interest might be larger or smaller than a single coil, therefore, the distance between any two coils should be considered accordingly. Also, shielding interconnects with the topmost metal layer reduces any unwanted or unintended forces to act on the object of interest while being manipulated by neighboring coils. Finally, distributing the coils across the array symmetrically helps in distributing the heat generated evenly by the coils.

\section{Biocompatibility}

Silicon nitride is used as a passivation layer on the surface of CMOS chips. Manipulation of cell cultures or maneuvering blood cells on the surface necessitates a suitable environment for cell growth or proper binding. If silicon nitride is not compatible with the object of interest, it can be etched out [6] or it can be coated with a biocompatible material like Parylene-C [7]. The thickness of the passivation layer must be carefully adjusted in order to have an effective force acting on, e.g., the magnetic (or magnetized) bead.

\section{COMPONENTS}

\section{A. Actuation and Steering (magnetic bead-labeled) elements}

The following modeling considers the magnetized force interaction when a magnetized object of interest (bead) is initially magnetized by a first coil on the array, which then is switched off, followed by a neighboring second coil switching on. Let the induced magnetic moment of the (magnetic) bead by the first coil is given as $M_{k}-1=\frac{1}{\mu_{0}} \chi V B_{k}-1$, where (the physical constants) $\mu_{0}, \chi, V$ are respectively the permeability, the susceptibility and volume of the bead due to the spatial coil located at $k-1$ [4]. Thus the generated magnetic potential for a bead which acquired the moment of magnetic field generated from location $k-1$ and now is acted upon by spatial coil location $k$ is given as the vector dot product of the 2 adjacent magnetic fields.

$$
\begin{aligned}
U & =-\frac{1}{2} M_{k-1} \cdot B_{k} \\
& =-\frac{1}{2}\left(\frac{1}{\mu_{0}} \chi V B_{k-1}\right) \cdot B_{k} .
\end{aligned}
$$

As the forces from the spatial coils are generated sequentially, it is thus assumed that the field $B_{k-1}$ is in steady state when the new field $B_{k}$ is generated from the adjacent coil $k$. Thus, the translational dynamics of the bead is given as [3], [4]

$$
\begin{aligned}
m \ddot{x} & =F_{d}+F_{m} \\
& \left.=\left(-6 a \pi \eta r_{B}\left(\dot{x}-v_{f}\right)\right)+\frac{1}{2} \frac{1}{\mu_{0}} \chi V\left(\vec{B}_{k-1}\right) \vec{B}_{k}\right)
\end{aligned}
$$

We relegate the definition of the (constant, physical) parameters to [4]. For the purpose of control, the equations are expressed in the compact form

$$
m \ddot{x}=\Gamma-d \dot{x}+b\left(\nabla \vec{B}_{k}\right) \vec{B}_{k-1},
$$

where $\Gamma$ is a vector determined by the fluid flow, $d$ is a (damping constant) determined by the flow friction to the bead, and the scalar $b$ is signifying scaling of the magnetic force generated. The rotational dynamics of the magnetized bead is expressed compactly in the form

$$
I \ddot{\theta}=N_{d}+N_{m}=-d_{r}(\dot{\theta}+\beta \dot{x})+\left(B_{k-1} \times B_{k}\right),
$$

where the parameters are compressed physical constants (see [4] and the references therein). It is known (theoretically and experimentally) that the rotational dynamics are fast in comparison to the translational dynamics and are often taken to be in the steady state of the induced moments as the bead quickly lines up with the adjacent magnetic field of spatial coil $k$. As an initial step for analysis, we focus on the translation dynamics with the rotational dynamics already assumed in the steady state.

The energy associated with the translational dynamics are the sum of the potential and kinetic energies as

$$
W(x, \dot{x})=\frac{1}{2}\left(\dot{x}^{T} \dot{x}\right)-\Gamma^{T} x+U
$$

The control signal is the generated magnetic field $B_{k}$ which is radiating from the spatial coil $k$ and is controlled by the current strength in the coil $k$. It is noted that the presence of fluidic flow renders the vector $\Gamma$ non-zero, and with the consideration of the inertia of the bead, the dynamics are non-gradient. Thus methods that exploit the gradient in devising control are not applicable. However, in practice, when the mass of the bead is neglected, the dynamics become gradients and the bead can be steered by the potential surface shaping. The platform array in effect discretize the potential surface spatially and the coil elements of the array define spatial regions of the potential field. Thus, an object can be steered along arbitrary paths along the platform surface. The energy function in (8) captures the dynamics of the physical system with control and thus represents the constraint in an optimization setting. In the optimization, one can derive the control sequence that would, e.g., steer a single or a population of beads to form a prescribed probability distribution or profile.

\section{B. Magnetic Flux Density and Force Simulations}

Each on-chip coil actuator in the array was designed to generate forces up to $6 \mathrm{nN}$ using a maximum current of up to $1.5 \mathrm{~mA}$. The direction of the force follows the right hand 


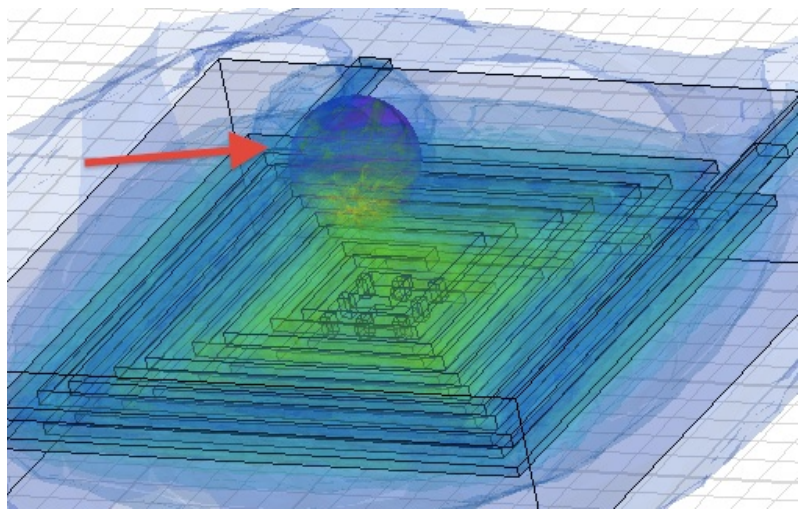

Fig. 3. Finite element simulation of the force generated on a magnetic bead. The red arrow points at the bead.

rule depending on the direction of the current passing through the coil. A square shape spiraling inductor was adopted in this design. The coil has the following characteristics: its resistance is $23.94 \mathrm{Ohms}$, its DC Inductance is $1.125 \mathrm{nH}$, its Length is $30 \mu \mathrm{m}$, its Metal Width is $1.8 \mu \mathrm{m}$, its Metal Spacing $1.2 \mu \mathrm{m}$, and the number of turns is 5. Using a Finite-ElementMethod simulator, the force generated on a magnetic bead using this coil, when a current of $1.5 \mathrm{~mA}$ passing through it, is $6.0354 \mathrm{nN}$. Figure 3 shows a 3D-view of the coil, the bead, and the magnetic flux density (the blue color is the weakest and the green color is the strongest field level).

The forces generated are maximized at the center of the coil. However, due to the fact that the coil is not a perfect spiral (a square in our case), the center of the coil will have two maxima as shown in figure 4 . The $\mathrm{X}$-Axis represents the distance from the center of the $30 \mu \mathrm{m} \times 30 \mu \mathrm{m}$ coil, the Y-Axis is the magnitude of the magnetic flux density (B), the colored curves are the magnitudes of (B) at a certain height from the topmost metal layer (not the passivation). Finally, to alleviate having two maxima, one can apply a $2 \mu \mathrm{m}$ silicon oxide and silicon nitride layers which also serve as a protection layer for the platform.

\section{Fabrication and Packaging}

This example design has been fabricated in $0.5 \mu \mathrm{m}$ standard CMOS. Figure 5 depicts one of three arrays integrated on the same $1.5 \mathrm{~mm} \times 1.5 \mathrm{~mm}$ die. The power consumption of the entire system is $9 \mathrm{~mW}$ using a $5 \mathrm{v}$ supply. The die was packaged and wire-bonded on an Printed Circuit Board (PCB) probe, similar to NeuroNexus A-series, which allowed us to submerge the whole design in liquid while still monitoring it under the microscope. The bonding wires were insulated with EpoxyTechnology $353 \mathrm{ND}$ to protect from circuit-shorting or damage.

\section{CONCLUSion}

The paper describes some example efforts towards the development of non-contact sensing and actuation using electromagnetic coil arrays in standard silicon technology. The silicon substrate serves as a platform for interacting with micro to nano scale particles on its surface. As well,

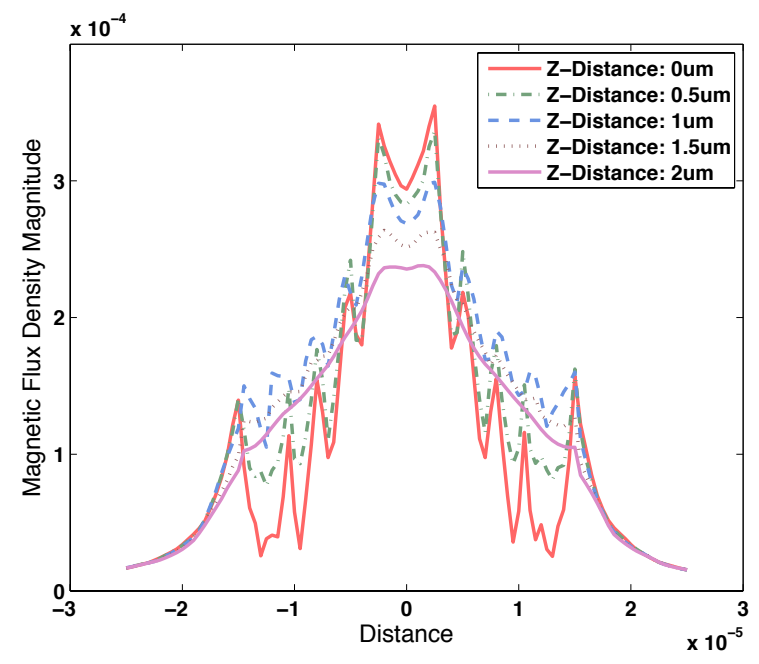

Fig. 4. The magnitude of the magnetic flux density generated by a 30x30 coil using $1.5 \mathrm{~mA}$ at different heights.

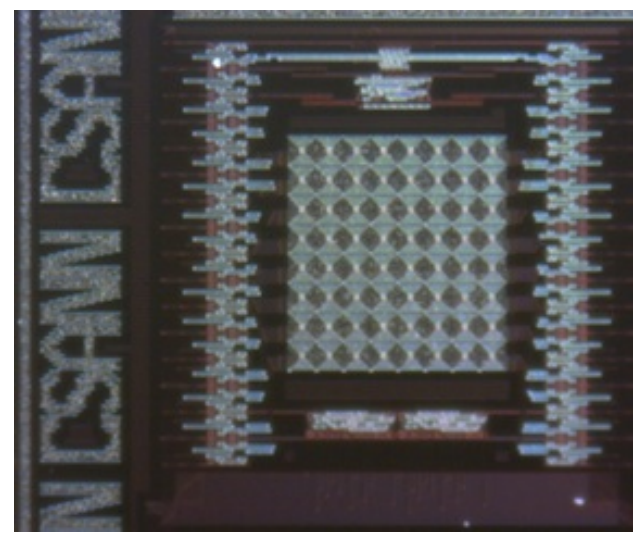

Fig. 5. Micrograph of one of the arrays implement in $0.5 \mu \mathrm{m}$ 3-metal-2poly-CMOS

it interfaces efficiently with existing computing and display devices.

\section{REFERENCES}

[1] F. Abu-Nimeh and F. Salem, "Integrated magnetic array for bioobject sensing and manipulation," Biomedical Circuits and Systems Conference, 2010. BioCAS 2010. IEEE, Nov 2010.

[2] W. Altman, J. Moreland, S. Russek, and V. Bright, "Optimization of spin-valve parameters for magnetic bead trapping and manipulation," Journal of Magnetism and Magnetic Materials, vol. 322, no. 21, pp. 3236-3239, 2010.

[3] U. Lehmann, M. Sergio, E. Dupont, and E. Labonne, "Actuation and detection of magnetic microparticles in a bioanalytical microsystem with integrated cmos chip," Nanosystems Design and Technology, Jan 2009.

[4] H. Lee, D. Ham, and R. Westervelt, CMOS biotechnology. SpringerVerlag New York Inc, 2007.

[5] J. Ohayon and P. Tracqui, "Computation of adherent cell elasticity for critical cell-bead geometry in magnetic twisting experiments," Annals of biomedical engineering, Jan 2005.

[6] H. Jansen, H. Gardeniers, M. Boer, M. Elwenspoek, and J. Fluitman, "A survey on the reactive ion etching of silicon in microtechnology," Journal of micromechanics and microengineering, vol. 6, p. 14, 1996.

[7] T. Chang, V. Yadav, S. De Leo, A. Mohedas, B. Rajalingam, C. Chen, S. Selvarasah, M. Dokmeci, and A. Khademhosseini, "Cell and protein compatibility of parylene-C surfaces," Langmuir, vol. 23, no. 23, pp. 11718-11 725, 2007. 